\title{
Finite Element Modeling of 3D Human Mesenchymal Stem Cell- Seeded Collagen Matrices Exposed to Tensile Strain
}

\author{
T. Wayne Pfeiler ${ }^{1}$, Ruwan D. Sumanasinghe ${ }^{1}$, and Elizabeth G. Loboa ${ }^{1, *}$ \\ Elizabeth G. Loboa: egloboa@ncsu.edu \\ ${ }^{1}$ Joint Department of Biomedical Engineering at North Carolina State, University and University of \\ North Carolina - Chapel Hill
}

\section{Abstract}

The use of human mesenchymal stem cells (hMSCs) in tissue engineering is attractive due to their ability to extensively self-replicate and differentiate into a multitude of cell lineages. It has been experimentally established that hMSCs are influenced by chemical and mechanical signals. However, the combined chemical and mechanical in vitro culture conditions that lead to functional tissue require greater understanding. In this study, finite element models were created to evaluate the local loading conditions on bone marrow derived hMSCs seeded in three dimensional collagen matrices exposed to cyclic tensile strain. Mechanical property and geometry data used in the models were obtained experimentally from a previous study in our laboratory and from mechanical testing. Eight finite element models were created to simulate three-dimensional hMSC-seeded collagen matrices exposed to different levels of cyclic tensile strain (10\% and $12 \%$ ), culture media (complete growth and osteogenic differentiating), and durations of culture (7 and 14 days). Through finite element analysis, it was determined that globally applied uniaxial tensile strains of $10 \%$ and $12 \%$ resulted in local strains up to $18.3 \%$ and $21.8 \%$, respectively. Model results were also compared to experimental studies in an attempt to explain observed differences between hMSC response to $10 \%$ and $12 \%$ cyclic tensile strain.

\section{Keywords}

Uniaxial Tensile Strain; hMSCs; Collagen; Bioreactor; Finite Element Modeling

\section{Introduction}

Functional bone tissue engineering for large critical defects remains an elusive goal in restorative medicine. Key to in vitro generation of nascent tissue with appropriate material properties for withstanding in vivo loading are the combined mechanical and chemical stimuli used to culture cells. Previous studies have shown that mechanical loading induces differentiation of human mesenchymal stem cells (hMSCs) and mesenchymal tissue into tissues such as bone, fibrous tissue, cartilage, and smooth muscle cells (Ignatius 2004, Altman 2002, Campbell 2006, Carter 1998, Kelly 2005, Park 2004). We have previously shown that cyclic tensile strain, even in the absence of osteogenic differentiating medium (i.e. hMSCs maintained in growth medium alone), promotes osteogenic differentiation of

\footnotetext{
(C) 2008 Elsevier Ltd. All rights reserved.

*Corresponding author. Tel.: +1 9195134015.
}

Publisher's Disclaimer: This is a PDF file of an unedited manuscript that has been accepted for publication. As a service to our customers we are providing this early version of the manuscript. The manuscript will undergo copyediting, typesetting, and review of the resulting proof before it is published in its final citable form. Please note that during the production process errors may be discovered which could affect the content, and all legal disclaimers that apply to the journal pertain. 
bone marrow-derived hMSCs when seeded in 3D type I collagen matrices (Sumanasinghe 2006). The cyclic tensile strains that were applied to hMSCs in vitro in that study (10\% and $12 \%)$, however, were much greater than the strains bone typically experiences under physiological conditions (0.09\% to 0.21\%) (Mikić 1995, Hillam 1996, Burr 1996).

This study focused on engineered three dimensional hMSC-seeded collagen constructs cultured in TissueTrain (Flexcell Int. Hillsborough, NC) culture plates. The TissueTrain system has been used in previous studies to impart mechanical stimuli to a variety of cells in three-dimensional culture (Garvin 2003, Qi 2006), and to investigate the effects of cyclic tensile strain on osteogenic differentiation of hMSCs. In brief, the system works by drawing a vacuum against a rubber membrane, which in turn pulls two anchors in tension. Between the anchors, a linear cell-seeded collagen matrix is created so that cyclic uniaxial tension may be applied (Fig. 1).

A previous study in our laboratory examined bone morphogenetic protein-2 (BMP-2) expression in $10 \%$ and $12 \%$ cyclically strained 3D hMSC-seeded collagen matrices at 7 and 14 days as compared to unstrained controls using the Tissue Train system. The results of that study showed that matrices exposed to cyclic tensile strain increased BMP-2 expression in the absence of osteogenic growth supplements; however differences existed between apparently similar $10 \%$ and $12 \%$ strain levels with $10 \%$ cyclic tensile strain leading to a greater increase in BMP-2 expression than 12\% cyclic tensile strain (Sumanasinghe 2006 A).

As a biomaterial and engineered tissue scaffold, collagen has been modeled in numerous finite element analysis (FEA) studies. Two-dimensional FEA has been used to quantify forces exerted by corneal fibroblasts on a surrounding collagen matrix (Roy 1999). FEA has also been used to predict differentiation and repair of cartilage surrounding injuries (Cullinane 2003). Two-dimensional models have been created to simulate extension of prealigned collagen gels. (Chandran 2007). Collagen fiber alignment has been predicted during compaction in cell-seeded tissue equivalents (Ohsumi 2008). To our knowledge, no previous study has examined the local stress and strains within cell-seeded collagen gel matrices cultured under cyclic tensile strain using three dimensional FEA.

The goal of the present study was to use finite element analysis to simulate the hMSCseeded collagen matrices under the mechanical loading conditions in which they were grown in culture. We hypothesized that local strains experienced by cells within the matrices would differ from the global strains applied by the bioreactor, and these local strains would change with time in culture. To investigate this hypothesis, we created finite element models of the three-dimensional hMSC-seeded collagen gels in an attempt to answer the following questions: 1 . What are the local stress and strain fields experienced by cells within the threedimensional collagen gels? 2. How do the local stress and strain fields vary at different time points in culture? 3. What may be the difference between $10 \%$ and $12 \%$ applied global cyclic tensile strain that causes a significant difference in expression of BMP-2?

\section{Methods}

\section{Mechanical Testing}

Eleven nonwoven Nylon mesh specimens (Cerex Advanced Fabrics Cantonment, FL) were tested in tension to determine the elastic modulus of the Cerex anchor of the TissueTrain (Flexcell Int., Hillsborough, NC) device (Fig. 1B). The mesh material consists of spunbond Nylon $0.09 \mathrm{~mm}$ thick with a basis weight of 0.60 ounce per square yard which was stamp cut to dog bone shaped test specimens. The cross sectional area of each sample was determined by averaging measured width at three locations and multiplying it by the 
manufacturer-provided thickness. Previously unstrained specimens were soaked in phosphate buffered saline (PBS) for one hour then tested in tension using an EnduraTEC ELF 3200 (Bose, Minnetonka, MN) under ramp displacement control at $0.05 \mathrm{~mm} / \mathrm{sec}(0.2 \%$ strain/sec) to $150 \%$ of maximum strain experienced by constructs when strained within the TissueTrain bioreactor. A 500 gram load cell was used to record the resulting forces. Elastic modulus was calculated by averaging the stiffness data from the linear portions of the stressstrain histories for each specimen.

Linear elastic moduli data of unstrained, $10 \%$, and $12 \%$ axially strained hMSC-seeded collagen matrices cultured in either complete growth or osteogenic medium were previously determined experimentally after 7 or 14 days through similar mechanical testing (Sumanasinghe 2007). In brief, Young's moduli and failure stresses of the hMSC-seeded collagen constructs were determined one day after completing culture strain regimen by applying a $0.5 \%$ per second constant strain rate to a $10 \mathrm{~mm}$ central section of each matrix until failure using a $500 \mathrm{gram}$ load cell with a force resolution of $0.03 \mathrm{~g}$.

\section{Actin Staining}

At 14 days in culture, actin morphology and alignment was visualized by staining the hMSC-seeded collagen matrices with Alexa 594 phalloidin (Molecular Probes, Eugene, OR). Matrices were rinsed twice in PBS and fixed for 30 minutes with $10 \%$ formalin. After fixing, matrices were rinsed twice in PBS then incubated in buffer consisting of $0.2 \%$ Triton $\mathrm{X}-100$ with $0.5 \%$ bovine serum albumin in PBS for 30 minutes. Matrices were then stained with $1 \mathrm{ug} / \mathrm{ml}$ phalloidin in buffer for 20 minutes while protected from light. Following staining, matrices were rinsed twice with PBS and imaged at 10X magnification using fluorescent microscopy (Leica Microsystems Inc., Bannockburn, IL).

\section{Finite Element Modeling}

Previous studies have shown that mesenchymal tissue and type I collagen gel scaffolds initially have a linear elastic response under physiological loading frequencies (Loboa 2004, Roeder 2002). Therefore, the matrices were modeled as isotropic, linear elastic materials based on these studies and previous mechanical testing results from our laboratory (Sumanasinghe 2007).

In the present study, finite element models were designed and solved using ABAQUS CAE version 6.4-3 (ABAQUS, INC. Providence, RI). A total of eight axisymmetric models, each representing one-half of the hMSC-seeded collagen gel at different time points, strain levels, and in either osteogenic or growth medium (Fig. 2), were constructed using geometry and material property data obtained experimentally (Table 1) and a Poisson's ratio of 0.49 . Other studies have modeled type I collagen and hMSCs with Poisson's ratios near 0.49 or 0.50 (Simha 1999, Zhang 2005, Darling 2008). Models in the present study simulated hMSCseeded collagen gels cultured in either osteogenic differentiation or complete growth medium, and exposed to $10 \%$ or $12 \%$ cyclic tensile axial strain, after 7 or 14 days time in culture. The shaft of each matrix was approximated with an elliptical cross section using width and thickness data taken in a previous study (Table 1) (Sumanasinghe 2008). In that study, the width and thickness data were taken by imaging the constructs in two planes and using Scanflex image analysis software (Flexcell, Hillsborough, NC), and mechanical property data were determined using the same technique described above for nonwoven Nylon anchors. Average widths of the elliptical sections ranged from $0.386 \mathrm{~mm}$ to 0.850 $\mathrm{mm}$ and average thicknesses ranged from $0.221 \mathrm{~mm}$ to $0.442 \mathrm{~mm}$. The anchor-attachment zone of the hMSC-seeded collagen gel was modeled to follow the anchor geometry with a small surrounding thickness (Fig. 3). The shaft was joined to the anchor attachment zone by lofting. One anchor was created for each model using geometry measurements taken from a 
TissueTrain plate (Flexcell Int.). An embedding constraint was used to attach the anchor into the matrix. The model was loaded by constraining axial motion at the midsection and applying a $10 \%$ or $12 \%$ tensile deformation to the end of the anchor.

Each completed model consisted of approximately 15,000 hexahedral elements. A linear static solution was conducted and stress and strain fields were recorded for each model. The reaction forces on the anchors necessary to achieve each strain were also recorded.

\section{Results \\ Mechanical testing}

Eleven nonwoven nylon mesh specimens had an average stiffness of 16.3 MPa with a standard deviation of 3.6 MPa. All specimens exhibited linear stress-strain relationships to the applied displacements (Fig. 4). The data from one specimen were not included due to inadequate nylon fiber mesh density, which led to immediate rupture.

\section{Cytoskeleton Staining}

Representative images taken near the center of the hMSC-seeded collagen gels appeared to show increased number of actin fibers and more consistent actin organization in matrices globally strained at 10\% (Fig. 5A, 5C) compared to 12\% (Fig. 5B, 5D). Fiber alignment in the applied strain direction (horizontal) also appeared to be more consistent in matrices globally strained at $10 \%$.

\section{Finite element modeling}

Axial strain-The strain magnitudes were highest within the central contracted portion of the matrices, gradually decreasing with the increase in cross section to the anchor, and lowest in the portion overlapping the anchors (Fig. 6). All models indicated local strains higher than the applied global strains within the central portions of the matrices. Depending on the specimen type being modeled, the increase in local strain over global strain varied from $6.4 \%$ to $9.8 \%$. The greatest increase of $9.8 \%$ over applied strain was found in the 14 day matrix strained globally at $12 \%$ (local strain $=21.8 \%$ ) while cultured in complete growth media (Fig. 6A). The lowest increase of $6.4 \%$ over applied strain was found in the 7 day matrix globally strained at $10 \%$ (local strain $=16.4 \%$ ) cultured in osteogenic differentiation media (Fig 6B).

Axial Stress-Stress fields followed a similar distribution to the strain fields described above (Fig. 7). The stress experienced by the central portion of the matrices varied from $83.2 \mathrm{KPa}$ in the model representing a 7 day matrix globally strained at $10 \%$ cultured in complete growth medium (Fig. 7A) to $430 \mathrm{KPa}$ as indicated in the model of a 14 day matrix globally strained at $10 \%$ cultured in complete growth medium (Fig. 7A).

Axial displacement-Axial displacements were linear through the central contracted portions of the matrices (Fig. 8). As the model stiffness increased through the greater cross section near the anchor, the change in displacement decreased. The portions of each model where the collagen gel overlapped the anchors exhibited much stiffer behavior than the rest of the model.

Effect of culture duration-Axial strain increased only marginally within the central section of all models from 7-day to 14-day hMSC-seeded collagen matrices (Table 2). Axial stress within the central contracted portion increased in models of matrices cultured in complete growth medium from day 7 to day 14 (Table 2). The greatest increase was within the $10 \%$ globally strained matrices cultured in complete growth media. The lowest increase 
in stress was indicated by the models of the $12 \%$ globally strained matrices cultured in osteogenic differentiation media.

\section{Discussion}

In the present study, we created 3D finite element models to determine the local stress and strain fields experienced by hMSCs cultured in 3D collagen matrices and exposed to global cyclic tensile strains of $10 \%$ and $12 \%$. We have previously shown that these apparently similar magnitudes of cyclic tensile strain result in distinctly different responses by hMSCs in 3D collagen gels with respect to BMP-2 mRNA expression, matrix contraction, nuclear morphology and orientation, and tensile properties of hMSC-seeded collagen gels (Sumanasinghe 2006, 2007 2008,). In the present study, the local strains varied with axial location in all models. The greater stiffness of the nylon nonwoven anchors caused stress shielding of the surrounding portion of the more compliant hMSC-seeded collagen matrix, resulting in the portion between the anchor tips experiencing an increased strain.

The 14 day models yielded higher axial stresses than 7 day models representing equivalent global strains and medium types, although the change was less for specimens cultured in osteogenic differentiating media as compared to complete growth media. The increases in stress from 7 day models to 14 day models were due in large part to decreases in cross sectional area. The 14 day specimens cultured in complete growth media had smaller cross sectional areas than the 14 day specimens cultured in osteogenic differentiating media. The smaller matrix area contributed to the increased stress. The actin fibrillar network appeared to be more diffuse and have more densely concentrated aggregates in the $12 \%$ global strain matrices (Fig. 5B, 5D) compared to the $10 \%$ global strain matrices(Fig. 5A, 5C), which appear to have greater fibrillar density and alignment in the global strain direction. This difference may be due to actin cytoskeleton damage that appears to occur in the $12 \%$ global strain matrices (Fig. 5B, 5D). It is possible that local strains resulting from the $12 \%$ global strain may be above a threshold that the hMSCs can tolerate without damage, in light of the encouraging experimental results from the $10 \%$ global applied strain matrices, which exhibited a significantly higher fold change in expression of BMP-2 over unstrained controls (Sumanasinghe 2006). A connection between mechanical stretch and rat MSC F-actin filament arrangement has been previously reported (Qi 2005). Work by other investigators suggests that actin filament bundles affect overall cell mechanical properties (Deguchi 2006). Such a strain threshold would need to be confirmed with experimental analyses quantitating F-actin content and morphology.

Another large component affecting the local stress and strain fields was the effect the hMSCs had on cross section of the collagen matrix as a result of matrix contraction by the cells. As cells exert traction forces on their environment, the substrate or 3D matrix surrounding them is contracted (Harris 1987, Tranquillo 1992, Choquet 1997, Sumanasinghe 2008). With longer times in culture, the hMSCs continued to contract the collagen matrix, causing the volume, thickness, and width to decrease. In three of the eight models, the predicted axial stress experienced by matrices in culture was greater than the failure stress levels reported previously during mechanical testing (specifically 430,157, and $365 \mathrm{kPa}$ model stress as compared to 171,124 , and $195 \mathrm{kPa}$ failure stress for cell seeded collagen gels cultured in complete growth medium subjected to $10 \%$ strain for 7 days, and $12 \%$ axial for 7 and 14 days respectively) (Sumanasinghe 2007). This was likely due to variations in methods by which the failure stress was calculated in the previous experimental studies and this study. The previous experimental studies measured cross sectional area of the hMSCseeded collagen matrices in an unstrained condition. When mechanical testing was conducted, the engineering failure stress was calculated based on the area of the initial unstrained cross section. The finite element models created in this study decreased in cross 
section as strain was applied due to Poisson effects, and the axial stress reported was based on the changing geometry.

As illustrated by the difference between 7 day and 14 day time points (Table 2), the global applied strains of $10 \%$ and $12 \%$ did not create consistent stresses within hMSC-seeded collagen matrices throughout the culture duration. It is possible that the hMSCs deposited collagen or calcium within the matrix that might have caused the stiffness of the matrix to increase. Some studies have also shown mechanical strain increases stiffness of cell-seeded collagen gels (Shearn 2007, Sumanasinghe 2007) while another found no significant increase (Nirmalanandhan 2007). Mineralization was not verified in this study. In a bioreactor system without feedback such as the one investigated here, this straining method could lead to a decrease in the local strain experienced by cells. Another study found effective increases of $10 \%$ to $20 \%$ over prescribed strains on two-dimensional, biaxial BioFlex (Flexcell, Int) culture plates which use similar rubber membrane substrates (Thompson 2007).

A limitation of this study is that the mechanical property and geometry data used in the models were taken from bone marrow-derived hMSCs from a single donor. It is possible that other cell lines may exhibit different results, as the amount of mineral or collagen deposited by hMSCs within the matrices may vary. It should also be noted that this study modeled hMSC-seeded collagen matrices in the linear elastic range of displacement, due to the applied cyclic strain frequency of $1 \mathrm{~Hz}$. Another study used much longer loading durations and found viscoelastic properties of both cell-seeded and acellular type I collagen gels matrices when held in tension for one hour (Karamichos 2006). This difference in material response of collagen gels is expected, due to the significant difference between loading frequencies used in the two studies.

In this study, we simulated 3D hMSC-seeded collagen gel matrices through 3D finite element modeling. We applied geometry and material property data previously determined experimentally to create models of matrices at 7 and 14 days cultured in complete growth medium or osteogenic medium and subjected to $10 \%$ or $12 \%$ global tensile strain. These models were used to determine the local stresses and strains within the matrices at 7 and 14 days. We also suggest that damage to the actin fibrillar network may occur at strains above $10 \%$ which may inhibit expression hMSC of BMP-2 mRNA.

This study emphasizes the importance of understanding the relationship between global strains applied by a bioreactor and local strains experienced by cells in culture. Transitioning from one bioreactor system to another or between different cell types requires an understanding of this strain relationship in order to achieve desired results. As the use of mechanical stimulation becomes increasingly common to a multitude of cell types in culture, a more complete understanding of the effective loading applied to cells in culture should enhance experimental results.

\section{Acknowledgments}

The authors thank Al Banes for donation of Nylon nonwoven material and technical advice. The authors also thank Susan Bernacki, Vinay Swaminathan and Eric Sills for their technical advice. Funding was provided by NIH STTR Grant \# 1R41AR052268-01A1 (AJB, EGL), an NCBC MRG grant (EGL), and a Powe Faculty Enhancement Award (EGL). 


\section{References}

Altman GH, Horan RL, Martin I, Farhadi J, Stark PR, Volloch V, Richmond JC, Vunjak-Novakovic G, Kaplan DL. Cell differentiation by mechanical stress. FASEB J. 2002; 16(2):270-272. [PubMed: 11772952]

Burr DB, Milgrom C, Fyhrie D, Forwood M, Nyska M, Finestone A, Hoshaw S, Saiag E, Simkin A. In vivo measurement of human tibial strains during vigorous activity. Bone. 1996; 18:405-10. [PubMed: 8739897]

Campbell JJ, Lee DA, Bader DL. Dynamic compressive strain influences chondrogenic gene expression in human mesenchymal stem cells. Biorheology. 2006; 43:455-70. [PubMed: 16912417]

Carter DR, Beaupre GS, Giori NJ, Helms JA. Mechanobiology of skeletal regeneration. Clin Orthop Relat Res. 1998; 355(Suppl):S41-55. [PubMed: 9917625]

Chandran PL, Barocas VH. Deterministic material-based averaging theory model of collagen gel micromechanics. J Biomech Eng. 2007; 129(2):137-47. [PubMed: 17408318]

Choquet D, Felsenfeld DP, Sheetz MP. Extracellular matrix rigidity causes strengthening of integrincytoskeleton linkages. Cell. 1997; 88(1):39-48. [PubMed: 9019403]

Cullinane DM, Salisbury KT, Alkhiary Y, Eisenberg S, Gerstenfeld L, Einhorn TA. Effects of the local mechanical environment on vertebrate tissue differentiation during repair: does repair recapitulate development? J Exp Biol. 2003; 206(Pt 14):2459-71. [PubMed: 12796461]

Darling EM, Topel M, Zauscher S, Vail TP, Guilak F. Viscoelastic properties of human mesenchymally-derived stem cells and primary osteoblasts, chondrocytes, and adipocytes. J Biomech. 2008; 41(2):454-64. [PubMed: 17825308]

Deguchi S, Ohashi T, Sato M. Tensile properties of single stress fibers isolated from cultured vascular smooth muscle cells. J Biomech. 2006; 39(14):2603-10. [PubMed: 16216252]

Garvin J, Qi J, Maloney M, Banes AJ. Novel system for engineering bioartificial tendons and application of mechanical load. Tissue Eng. 2003; 9:967-79. [PubMed: 14633381]

Harris AK. Cell motility and the problem of anatomical homeostasis. J Cell Sci. 1987; 8(Suppl):12140.

Hillam R, Jackson M, Goodship A, Skerry T. Comparison of physiological strains in the human skull and tibia. Bone. 1996; 19:686-686.

Ignatius A, Blessing H, Liedert A, Kaspar D, Kreja L, Friemert B, Claes L. Effects of mechanical strain on human osteoblastic precursor cells in type I collagen matrices. Orthopade. 2004; 33:1386-93. Article in German. [PubMed: 15578256]

Karamichos D, Brown RA, Mudera V. Complex dependence of substrate stiffness and serum concentration on cell-force generation. J Biomed Mater Res A. 2006; 78:407-15. [PubMed: 16715519]

Kelly DJ, Prendergast PJ. Mechano-regulation of stem cell differentiation and tissue regeneration in osteochondral defects. J Biomech. 2005; 38:1413-1422. [PubMed: 15922752]

Loboa EG, Fang TD, Warren SM, Lindsey DP, Fong KD, Longaker MT, Carter DR. Mechanobiology of mandibular distraction osteogenesis: experimental analyses with a rat model. Bone. 2004; 34:336-343. [PubMed: 14962812]

Mikić B, Carter DR. Bone strain gage data and theoretical models of functional adaptation. J Biomech. 1995; 28(4):465-9. [PubMed: 7738056]

Nirmalanandhan VS, Dressler MR, Shearn JT, Juncosa-Melvin N, Rao M, Gooch C, Bradica G, Butler DL. Mechanical stimulation of tissue engineered tendon constructs: effect of scaffold materials. J Biomech Eng. 2007; 129:919-23. [PubMed: 18067397]

Ohsumi TK, Flaherty JE, Evans MC, Barocas VH. Three-dimensional simulation of anisotropic celldriven collagen gel compaction. Biomech Model Mechanobiol. 2008; 7(1):53-62. [PubMed: 17354006]

Park JS, Chu JS, Cheng C, Chen F, Chen D, Li S. Differential effects of equiaxial and uniaxial strain on mesenchymal stem cells. Biotechnol Bioeng. 2004; 88:359-68. [PubMed: 15486942]

Qi J, Chi L, Faber J, Koller B, Banes AJ. ATP reduces gel compaction in osteoblast-populated collagen gels. J Appl Physiol. 2006; 102:1152-60. [PubMed: 17122380] 
Qi MC, Hu J, Zou SJ, Han LC, Luo E. The changes of cytoskeleton F-actin in rat bone marrow mesenchymal stem cells and calvarial osteoblasts under mechanical strain. West China Journal of Stomatology. 2005; 23:110-2. [PubMed: 15952618]

Roeder BA, Kokini K, Sturgis JE, Robinson JP, Voytik-Harbin SL. Tensile mechanical properties of three-dimensional type I collagen extracellular matrices with varied microstructure. J Biomech Eng. 2002; 124:214-22. [PubMed: 12002131]

Roy P, Petroll WM, Chuong CJ, Cavanagh HD, Jester JV. Effect of cell migration on the maintenance of tension on a collagen matrix. Ann Biomed Eng. 1999; 27(6):721-30. [PubMed: 10625145]

Shearn JT, Juncosa-Melvin N, Boivin GP, Galloway MT, Goodwin W, Gooch C, Dunn MG, Butler DL. Mechanical stimulation of tendon tissue engineered constructs: effects on construct stiffness, repair biomechanics, and their correlation. J Biomech Eng. 2007; 129:848-54. [PubMed: 18067388]

Simha NK, Fedewa M, Leo PH, Lewis JL, Oegema T. A composites theory predicts the dependence of stiffness of cartilage culture tissues on collagen volume fraction. J Biomech. 1999; 32:503-509. [PubMed: 10327004]

Sumanasinghe RD, Bernacki SH, Loboa EG. Osteogenic Differentiation of Human Mesenchymal Stem Cells in Collagen Matrices: Effect of Uniaxial Cyclic Tensile Strain on Bone Morphogenetic Protein (BMP-2) mRNA Expression. Tissue Eng. 2006; 12(12):3459-65. [PubMed: 17518682]

Sumanasinghe RD, Loboa EG. Effects of Cyclic Tensile Strain on Osteogenesis and Tensile Properties of Human Mesenchymal Stem Cell Seeded Collagen Matrices. Annals of Biomed Eng. 2007 submitted.

Sumanasinghe RD, Osborne JA, Loboa EG. Mesenchymal stem cell seeded collagen matrices for bone repair: Effects of uniaxial cyclic tensile strain, cell density and media conditions on matrix contraction in vitro. J Biomed Mater Res A. 2008 accepted.

Thompson MS, Bleckwehl FH, Ott CE, Epari DR, Duda GN. Mechanical Characterisation of the BioFlex System. Tissue Eng. 2007; 13(6):1367-1392. [PubMed: 17579535]

Tranquillo RT, Durrani MA, Moon AG. Tissue engineering science: consequences of cell traction force. Cytotechnology. 1992; 10(3):225-50. [PubMed: 1369238]

Zhang J, Jin GC, Meng LB, Jian LH, Wang AY, Lu SB. Strain and mechanical behavior measurements of soft tissues with digital speckle method. J Biomed Opt. 2005 May-Jun;10(3):4854-57. 


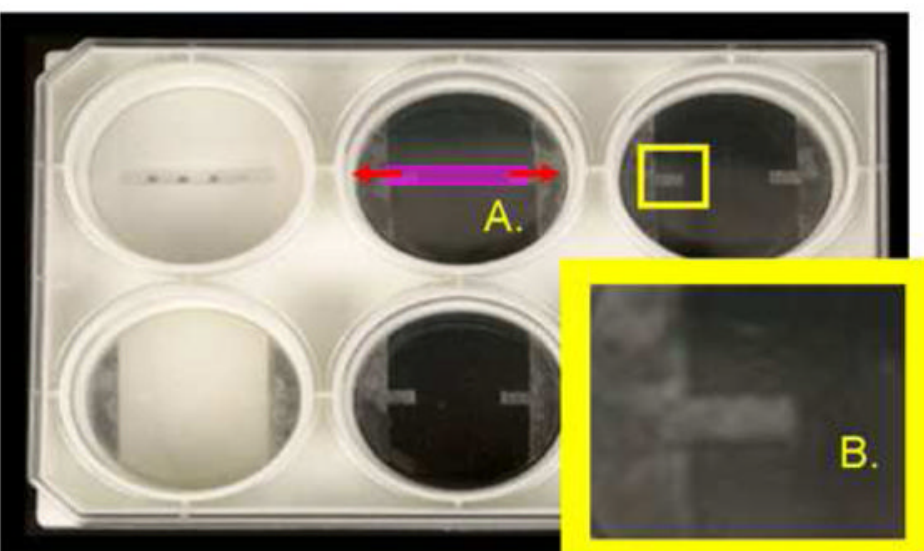

Figure 1.

TissueTrain culture plate: A. Illustration of MSC-seeded collagen gel set between anchors and direction of uniaxial tensile strain. B. Detail of nylon nonwoven anchor. 


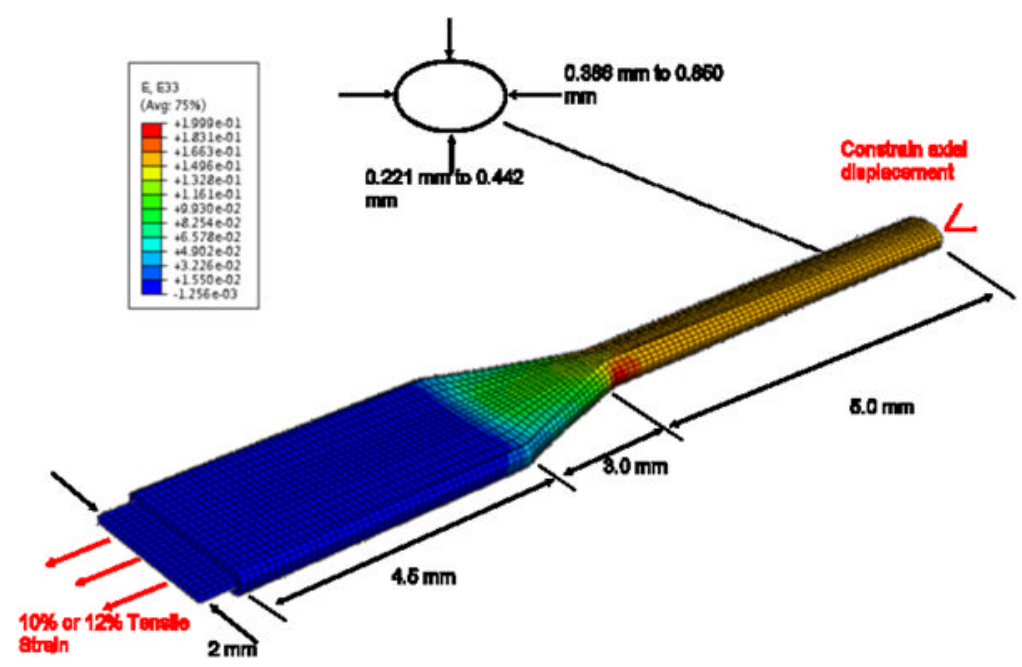

Figure 2.

Representative example of matrix geometry with embedded anchor and boundary conditions. Detail shows shaft cross section. Shading illustrates strain resulting from matrix loading. Model shown is globally strained at $10 \%$ at 7 days in culture with osteogenic differentiating medium. 


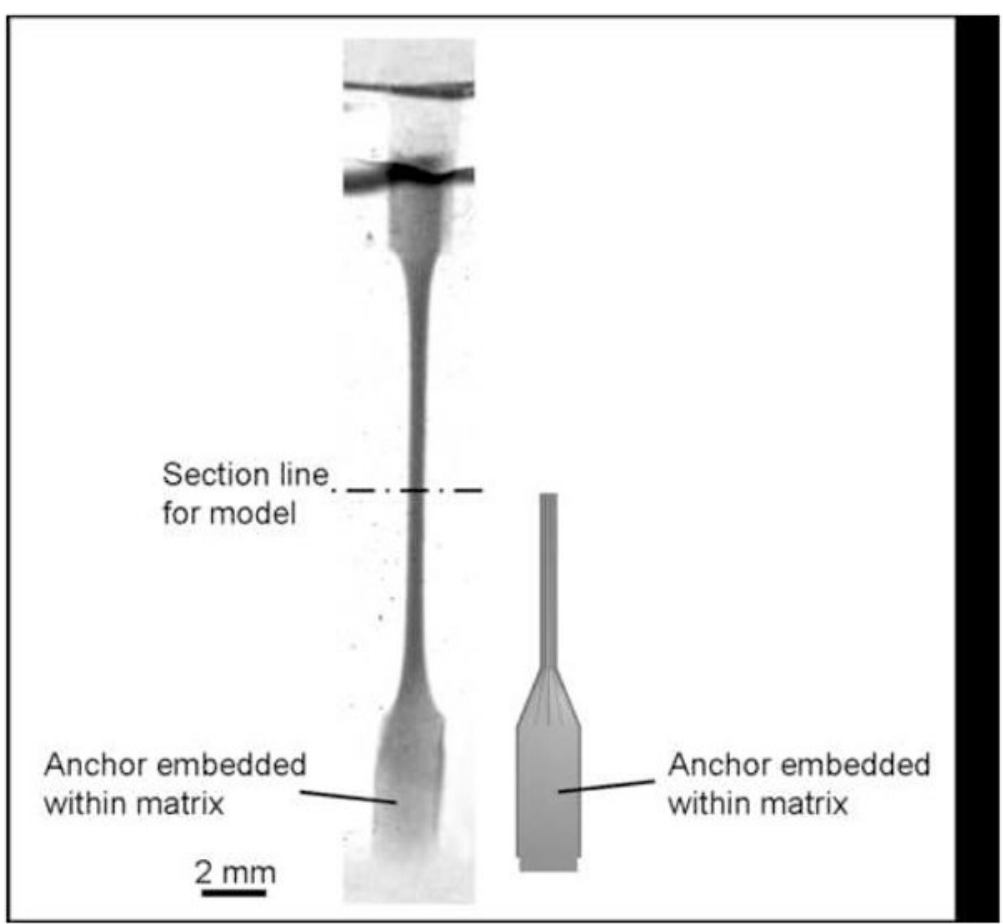

Figure 3.

Comparison of representative hMSC-seeded collagen matrix on left with axisymmetric finite element model on right. 


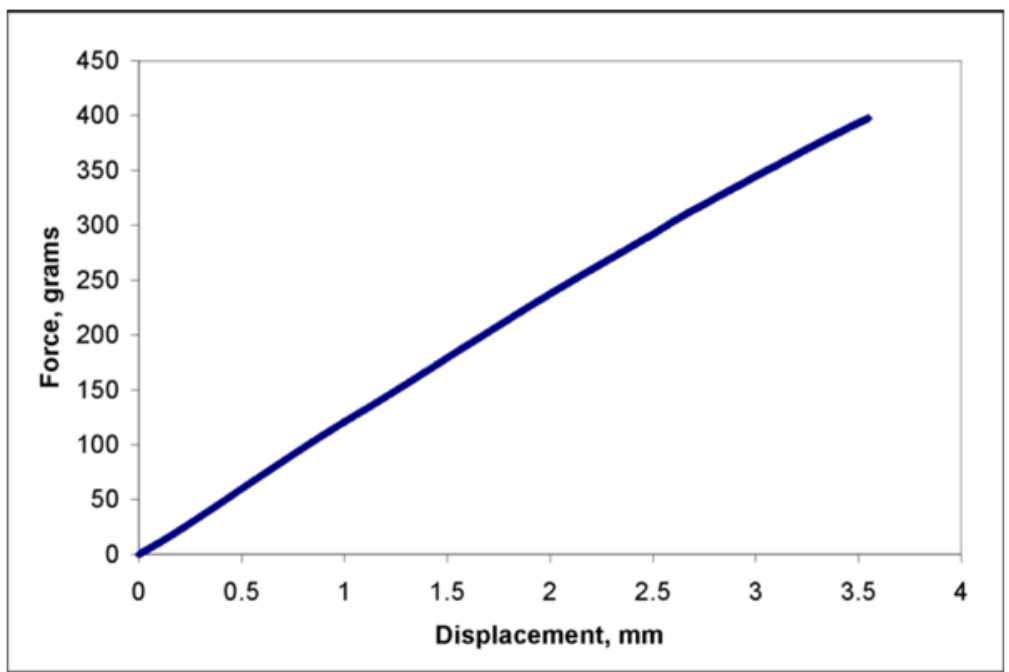

Figure 4.

Representative example of linear response to loading during mechanical testing of nylon nonwoven mesh anchors. 

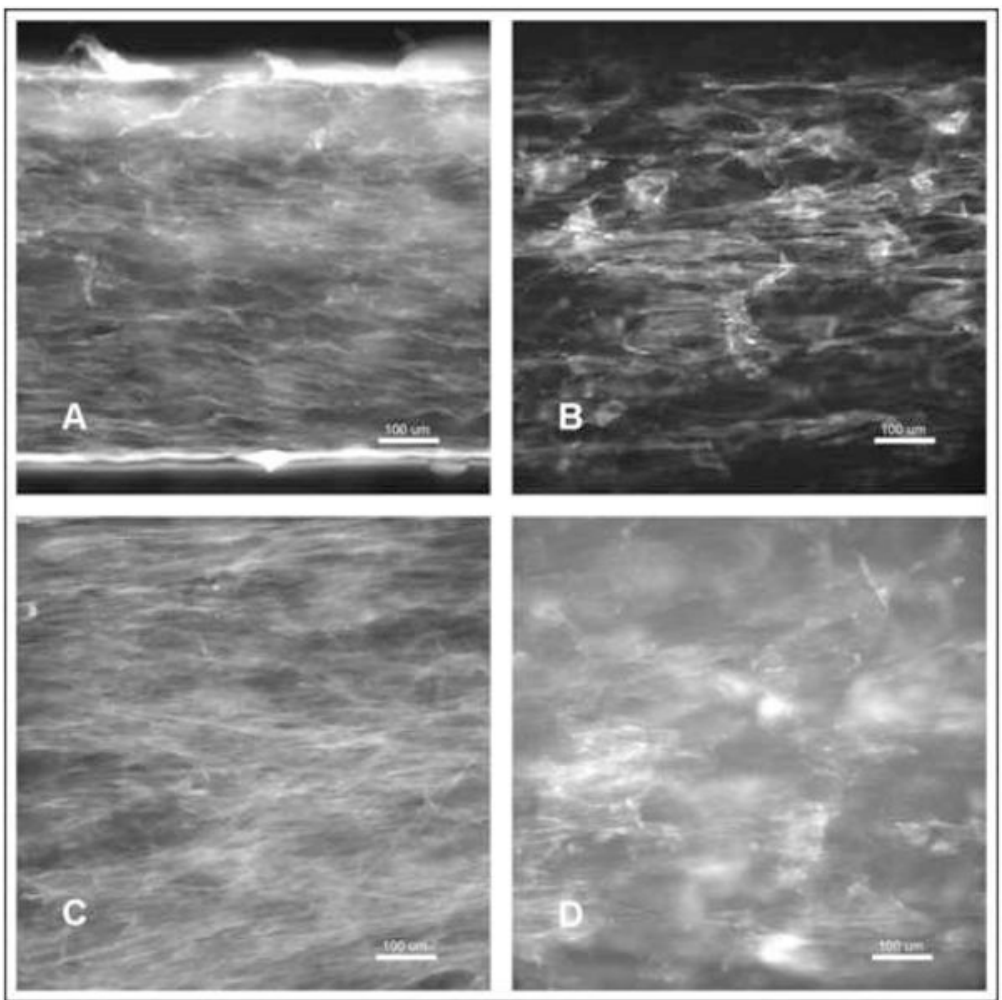

Figure 5.

Actin filaments within matrices stained with Alexa 594 phalloidin. Matrices globally strained at 10\% (A and C) and 12\% (B and D). Matrices cultured in complete growth medium (A and $\mathrm{B}$ ) and osteogenic differentiation medium (C and $\mathrm{D})$. 


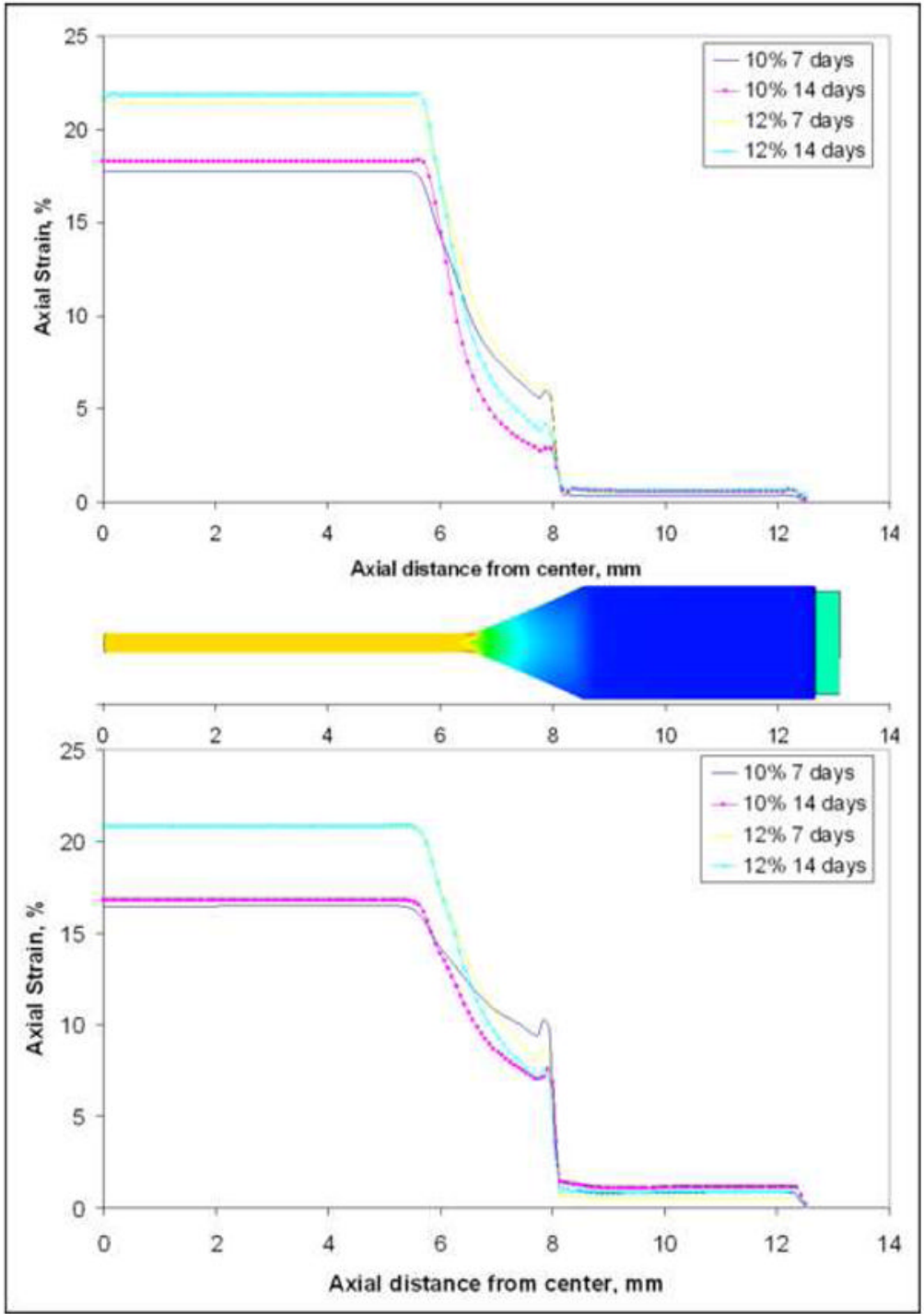

Figure 6.

Axial strain as a function of distance from center of matrices cultured in A) complete growth medium and B) osteogenic differentiating medium. 


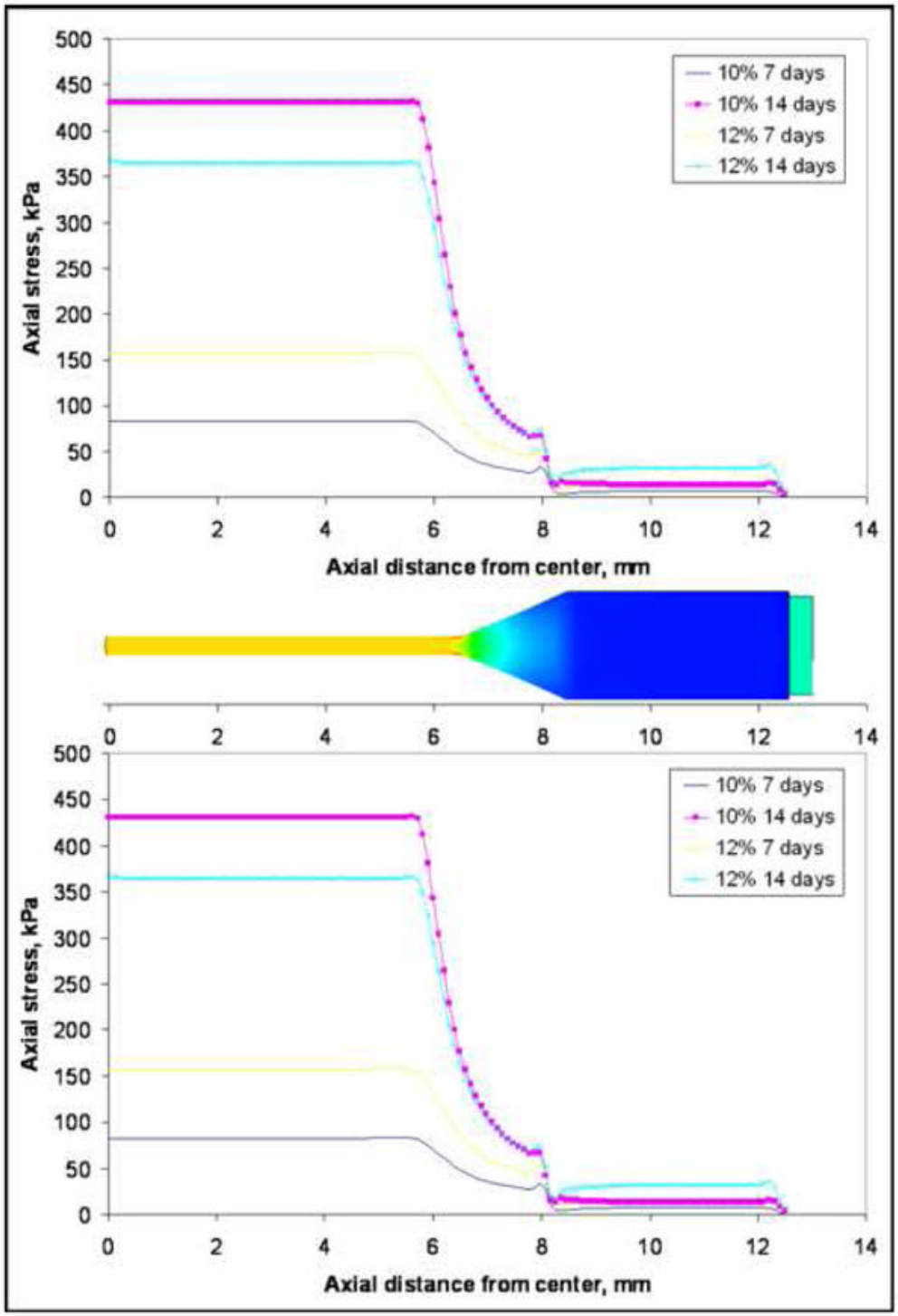

Figure 7.

Axial stress as a function of distance from center of matrices cultured in A) complete growth medium and B) osteogenic differentiating medium. 

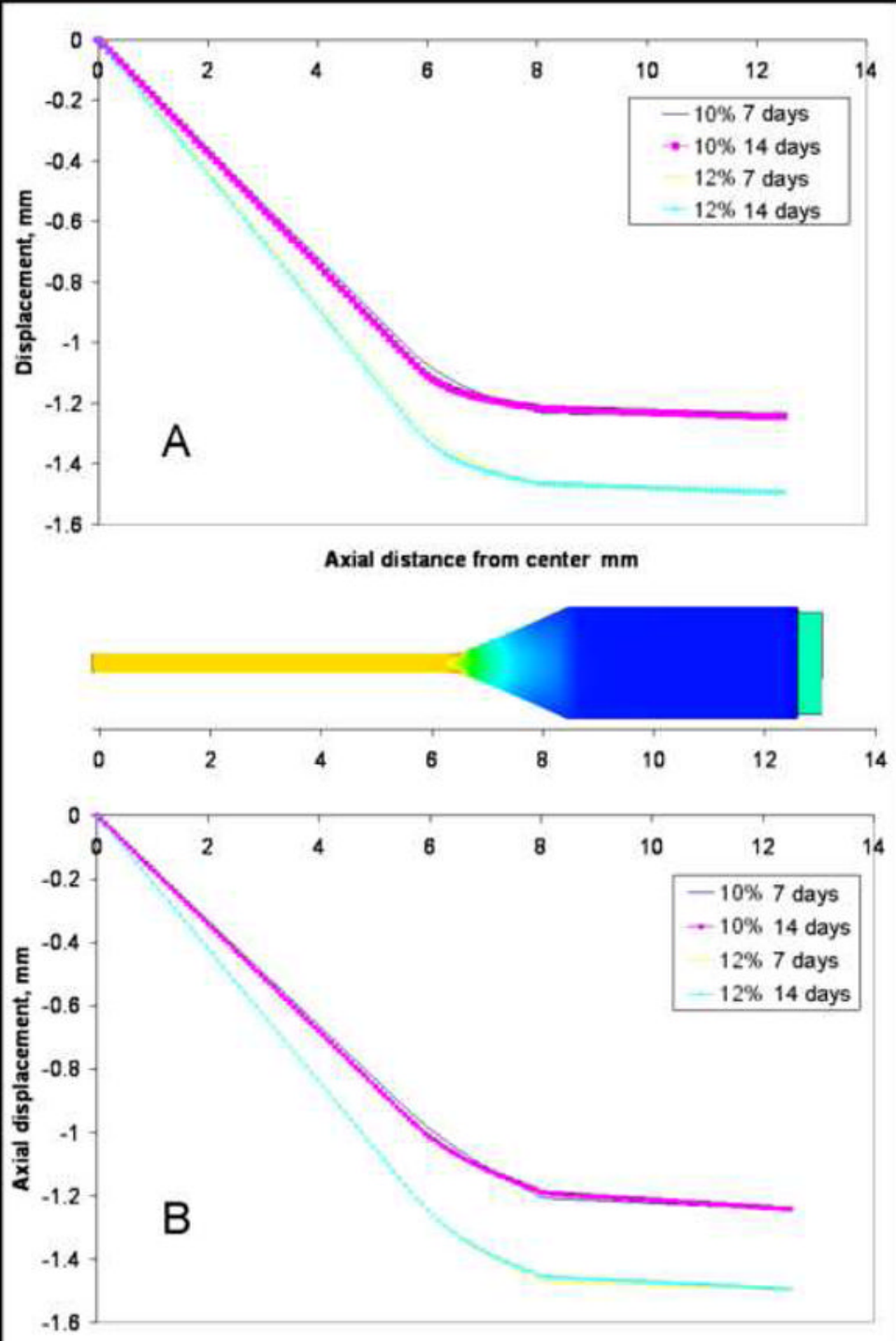

Axial distance from center, $\mathrm{mm}$

Figure 8.

Axial displacement as a function of distance from center of matrices cultured in A) complete growth medium and B) osteogenic differentiating medium. 


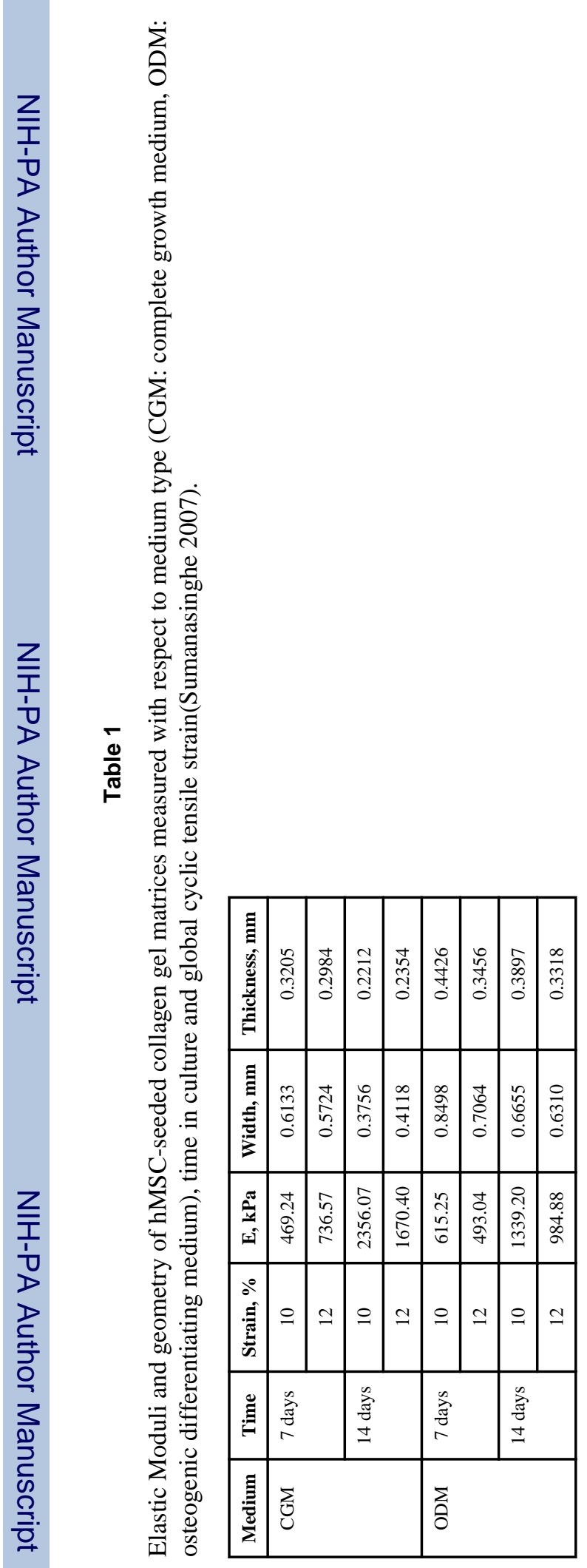

J Biomech. Author manuscript; available in PMC 2014 March 09. 


\section{Table 2}

Summary of finite element model results in center of cell-seeded construct by medium type, time in culture and global applied strain. CGM: Complete growth medium; ODM: Osteogenic differentiating medium.

\begin{tabular}{|c|c|c|c|c|}
\hline Medium & Time & $\begin{array}{c}\text { Global Applied Cyclic Tensile } \\
\text { Strain, \% }\end{array}$ & $\begin{array}{c}\text { Average Local Axial Strain, \% (St } \\
\text { Dev) }\end{array}$ & $\begin{array}{c}\text { Average Local Axial Stress, } \mathbf{k P a} \text {, } \\
\text { (St Dev) }\end{array}$ \\
\hline \multirow{2}{*}{ CGM } & \multirow{2}{*}{7 days } & 10 & $17.7(0.03)$ & $83.2(0.1)$ \\
\cline { 3 - 5 } & \multirow{2}{*}{14 days } & 12 & $21.4(0.01)$ & $157.5(0.3)$ \\
\cline { 3 - 5 } & & 10 & $18.3(0.02)$ & $430.4(0.1)$ \\
\hline \multirow{2}{*}{ ODM } & \multirow{2}{*}{7 days } & 12 & $21.8(0.04)$ & $365.1(0.5)$ \\
\cline { 3 - 5 } & & 10 & $16.4(0.08)$ & $101.2(0.3)$ \\
\cline { 2 - 5 } & \multirow{2}{*}{14 days } & 12 & $20.8(0.06)$ & $224.8(0.1)$ \\
\cline { 3 - 5 } & & 10 & $16.8(0.0003)$ & $102.6(0.2)$ \\
\hline
\end{tabular}

